PAPER

\title{
Quantitative assessment of driving performance in Parkinson's disease
}

\author{
J M Wood, C Worringham, G Kerr, K Mallon, P Silburn
}

See Editorial Commentary, page 159

J Neurol Neurosurg Psychiatry 2005;76:176-180. doi: 10.1136/jnnp.2004.047118

Objectives: The primary aim of this study was to determine how Parkinson's disease (PD) affects driving performance. It also examined whether changes in driver safety were related to specific clinical disease markers or an individual's self rating of driving ability.

See end of article for authors' affiliations .....................

Correspondence to: DrJ M Wood, School of Optometry, Queensland University of Technology, Victoria Park Road, Kelvin Grove Q4059, Australia; i.wood@qut.edu.au

Received 7 June 2004 In revised form 8 September 2004 Accepted 9 September 2004
Methods: The driving performance of 25 patients with idiopathic PD and 21 age matched controls was assessed on a standardised open road route by an occupational therapist and driving instructor, to provide overall safety ratings and specific driving error scores.

Results: The drivers with PD were rated as significantly less safe $(p<0.05)$ than controls, and more than half of the drivers with PD would not have passed a state based driving test. The driver safety ratings were more strongly related to disease duration $(r=-0.60)$ than to their on time Unified Parkinson's Disease Rating Scale $(r=-0.24)$. Drivers with PD made significantly more errors than the control group during manoeuvres that involved changing lanes and lane keeping, monitoring their blind spot, reversing, car parking, and traffic light controlled intersections. The driving instructor also had to intervene to avoid an incident significantly more often for drivers with PD than for controls. Interestingly, driver safety ratings were unrelated to an individual's rating of their own driving performance, and this was the case for all participants.

Conclusions: As a group, drivers with PD are less safe to drive than age matched controls. Standard clinical markers cannot reliably predict driver safety. Further studies are required to ascertain whether the identified driving difficulties can be ameliorated.
$\mathrm{P}$ arkinson's disease (PD) is a progressive neurodegenerative disease that affects motor and sometimes cognitive function, which could potentially impair driving performance, thereby limiting mobility and independence. Few studies have evaluated driving performance in PD. An exception has been the potential impact of medication induced sleep episodes on driving performance. ${ }^{1}$ However, these studies have methodological problems, ${ }^{2}$ and sleep episodes are arguably a less important source of driving difficulties than those that stem directly from the disease. ${ }^{3}$ For example, in addition to the cardinal symptoms of bradykinesia, tremor, and rigidity, several less obvious deficits include difficulties in movement planning, ${ }^{4}$ movement sequences, ${ }^{5}$ and simultaneous movements, ${ }^{6}$ in addition to subtle visual defects and impairments in shifting attentional set. ${ }^{7}$ Therefore, it is imperative to characterise fully the impact of PD on driving performance.

Madeley et al reported longer reaction times and reduced steering accuracy in a driving simulator in a small sample of patients with PD compared with controls. ${ }^{8}$ These impairments were significantly related to the PD group's Webster scores. Lings and Dupont ${ }^{9}$ also found increased reaction times and directional errors in PD using a simulator, but did not find a relation between the Webster scale and driving simulator performance. Recently Zesiewicz et al also used a simulator to assess PD driving performance; however, the only reported driving measure was the number of collisions, which was higher in those with PD than in control drivers, and was related to both the Hoehn and Yahr scale and the Unified Parkinson's Disease Rating Scale (UPDRS). ${ }^{10}$ Despite the known limitations of simulators, ${ }^{11}$ all of these studies demonstrated poorer driving skills in the individuals with PD compared with controls.

Although no studies have dealt with state reported crash rates for PD, Dubinsky and colleagues ${ }^{12}$ found that crashes (self reported retrospectively over three years) were more common in drivers with PD than in controls, and were related both to the Hoehn and Yahr rating and to dementia (Mini Mental State Examination; MMSE). However, the relation between self reported crash rates and state registered crashes has proved to be inconsistent. ${ }^{13}$

Only two studies, undertaken in Scandinavia ${ }^{14}$ and the $\mathrm{UK},{ }^{15}$ have assessed the on road driving performance of drivers with PD. Heikkilä et al reported that drivers with PD were significantly less safe than controls, with $35 \%$ rated as being unsafe to drive, and reported a low level of agreement between safety ratings of the neurologist and the driving instructor, compared with safety self ratings of drivers with PD. ${ }^{14}$ A combination of slowed visual processing, levodopa dosage, and age was shown to explain $67 \%$ of the variance in driving test scores in drivers with PD. ${ }^{14}$ Recently, Radford et al assessed drivers with PD on the open road, but included no control group. ${ }^{15}$ In contrast to Heikkilä et al, ${ }^{14}$ they found that only six of 33 drivers with PD were rated as unsafe, but this discordant finding may have resulted from selection bias, because participants were volunteers rather than a consecutive patient series. The use of a control group recruited in the same manner and from the same community groups assumes greater importance in such situations. Like Madeley et al, they found a link between the Webster scale and driving performance, although the small number of unsafe drivers in the sample limits this finding's validity.

Our present study investigated the effect of PD on overall driving safety and specific aspects of driving performance on the open road, using well established, quantitative scoring methods. A further aim was to determine whether any

Abbreviations: MMSE, Mini Mental State Examination; PD, Parkinson's disease; UPDRS, Unified Parkinson's Disease Rating Scale 
Table 1 Group mean (SD) of the characteristics of the participant groups

\begin{tabular}{lll}
\hline & Parkinson's disease & Control participants \\
\hline Age (years) & $63.7(6.8)$ & $65.2(8.6)$ \\
UPDRS score & $27.4(11.3)$ & \\
Hoehn and Yahr & $2.3(0.7)$ & \\
Disease duration (years) & $6.2(4.6)$ & \\
Mean levodopa dosage & $672.6(512.5)$ \\
(mg) & \\
\hline \multicolumn{2}{l}{ UPDRS, Unified Parkinson's Disease Rating Scale. }
\end{tabular}

changes in driver safety could be predicted by clinical disease markers or an individual's rating of their own driving ability.

\section{METHODS}

\section{Participants}

Twenty five participants (2l men, four women) with a diagnosis of idiopathic PD and 21 control participants (18 men, three women) in good general health, who drove at least one day each week, performed an on road driving assessment. All participants scored 24 or more on the MMSE, ${ }^{16}$ and none was excluded on the basis of dementia. All participants were community dwelling volunteers who had attended presentations at PD support group meetings. The clinical indicators recorded for participants with PD from the participating neurologist included time since the onset of PD symptoms, on time Hoehn and Yahr score, UPDRS (subscales and overall scale), and current levodopa dosage where available. Four of the participants were taking artane, and three of these patients were also taking levodopa medications. There was no significant difference between the safety ratings of those participants with PD who were taking artane and those not on this medication. The mean age of the participants with PD and the controls did not differ significantly $\left(\mathrm{t}_{44}=0.67 ; \mathrm{p}=0.51\right)$. All testing (driving and laboratory based) was undertaken when the participants were optimally medicated. Table 1 summarises the group characteristics.

Our study was conducted in accordance with the requirements of the Queensland University of Technology human research ethics committee. All participants were given a full explanation of the experimental procedures and written informed consent was obtained, with the option to withdraw from the study at any time.

To obtain an overall sense of the driving characteristics and perceptions of the participants, a previously validated 57 item

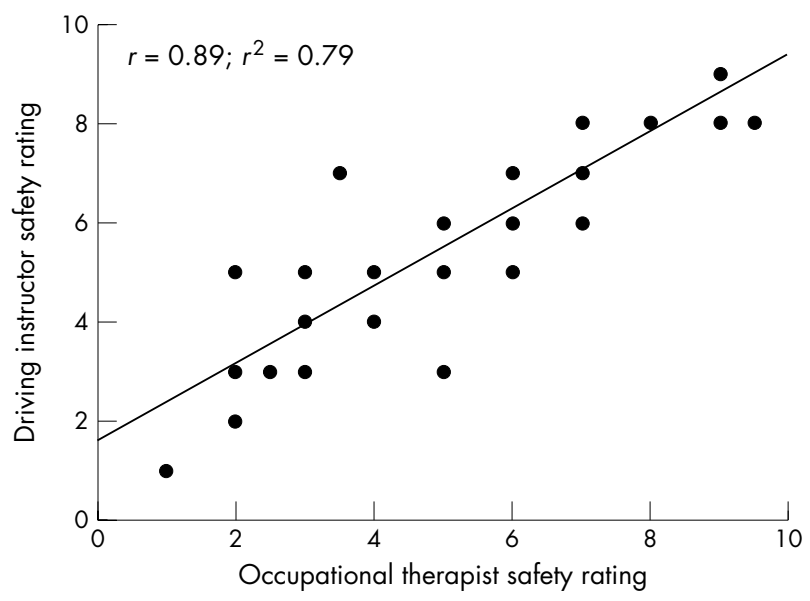

Figure 1 Correlation between the global driver safety ratings for all participants assigned by the occupational therapist and the driving instructor. The figure includes many overlying points. questionnaire was administered. ${ }^{17}$ Questions related to driving experience and habits, perceived difficulty (on a scale of 1 (quite difficult) to 5 (quite easy)) for 24 specific situations, and perceived difficulty (on the same 5 point scale) for 12 specific tasks. The questionnaire also included items relevant to PD, such as problems with moving the foot from one pedal to another.

\section{Driving performance}

Driving performance was measured in an automatic, dual brake vehicle under in traffic conditions using a previously validated technique. ${ }^{18} 19$ An accredited professional driving instructor, experienced in assessment, sat in the front passenger seat and was responsible for maintaining vehicle safety. A driver trained occupational therapist, experienced in the assessment of driving and rehabilitation, sat in the rear seat. Both assessed driving safety independently, using well defined criteria. Neither was aware of the categorisation of the participants.

All participants were directed to drive along the same $19.4 \mathrm{~km}$ open road route that contained a range of typical driving situations. Seventy per cent of the driving was under directed instruction and $30 \%$ was under self directed navigation, where the participant had to find their own way to a particular destination.

\section{Occupational therapist assessment}

The occupational therapist scored seven aspects of driving performance at 147 locations along the route (general observation, observation of blind spots, indication (signalling), braking/acceleration, lane positioning, gap selection, and approach). Failing any aspect of performance resulted in failure of the whole task for a given location, giving an overall score representing the percentage of locations where no errors were made. The number of each error type was also recorded. In addition, each of the 147 locations was allocated to one of nine categories, including roundabouts, merging, pulling in/pulling out, traffic light and non-traffic light controlled intersections, reversing and parking, one way and two way traffic, and lane changing. This allowed the identification of situations where drivers had the most difficulty.

The occupational therapist also gave an overall safety rating from 1 to 10 , according to current transport licensing standards. A score between 1 and 3 indicated that the instructor had to take action to avoid an incident, or that the driver hit a significant object and should consider ceasing

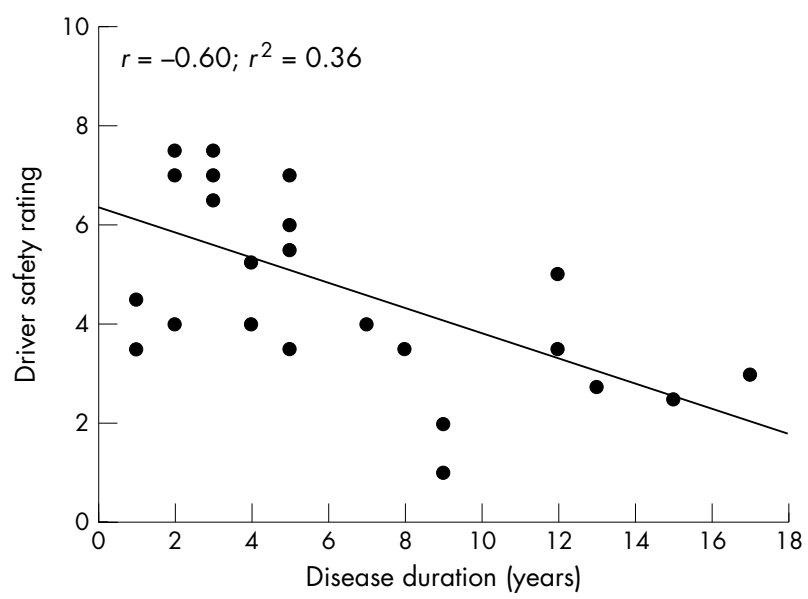

Figure 2 The association between the time since diagnosis of Parkinson's disease (PD) and average driver safety rating for each of the participants with PD. The figure includes some overlying points. 
Table 2 Overall driving score and types of driving errors made by the two groups as assessed by the occupational therapist

\begin{tabular}{|c|c|c|c|c|}
\hline \multirow[b]{2}{*}{ Driving scores } & \multicolumn{2}{|c|}{ Participant groups } & \multicolumn{2}{|l|}{ Statistics } \\
\hline & Controls & Parkinson's & t Value & p Value \\
\hline Overall score \% & $83.95(1.61)$ & $77.18(2.15)$ & 2.43 & 0.02 \\
\hline Critical errors & $0.38(0.17)$ & $1.12(0.36)$ & -1.75 & 0.09 \\
\hline Observation errors & $3.14(0.63)$ & $5.04(0.93)$ & -1.62 & 0.11 \\
\hline Blind spot errors & $5.19(0.66)$ & $7.52(0.74)$ & -2.30 & 0.03 \\
\hline Indicator errors & $6.09(0.58)$ & $6.80(0.77)$ & -0.71 & 0.48 \\
\hline Brake/accelerator errors & $8.19(1.26)$ & $9.80(1.76)$ & -0.72 & 0.48 \\
\hline Lane keeping errors & $4.62(1.00)$ & $10.20(1.62)$ & -2.80 & 0.01 \\
\hline Gap selection errors & $3.62(0.60)$ & $4.40(0.63)$ & -0.89 & 0.38 \\
\hline Approach errors & $8.28(1.24)$ & $10.36(1.57)$ & -1.00 & 0.32 \\
\hline Directed errors & $27.14(3.64)$ & $38.60(4.40)$ & -1.96 & 0.06 \\
\hline Self directed errors & $12.00(1.89)$ & $15.52(2.26)$ & -1.17 & 0.25 \\
\hline
\end{tabular}

driving. A score of 4 or 5 indicated poor driving and observation skills, whereas a score in the range of 6 to 8 indicated average driving skills, but with some bad habits. Finally, a score of 9 or 10 reflected good to excellent driving and observational skills.

\section{Driving instructor assessment}

The driving instructor provided an independent overall safety rating using the same scoring criteria as the occupational therapist. In addition, he recorded the number of critical and non-critical driving errors made, and the number of instructor interventions.

Participants rated the difficulty of the driving route and conditions compared with their typical driving (on a scale of $1-5$, where " $1=$ much easier" to " $5=$ much harder"), and their performance on the test compared with the typical driver (on a scale of $1-5$, where " $1=$ excellent" to "5 = poor").

\section{Data analysis}

The driving data were analysed using independent group $t$ tests to identify any group differences with directed or self directed navigational instruction, and were broken down into error types and locations. The familywise error rate was not corrected for because, as this is one of the first descriptions of driving error types and locations in PD, it was thought important not to miss potential problem areas by using unduly conservative methods. The safety ratings of the occupational therapist and driving instructor were compared to determine the level of inter-rater agreement, in addition to the association with disease markers (time since diagnosis, on time UPDRS and Hoehn and Yahr scale). For the driving history questionnaire, those questions for which participants used a scale from 1 to 5 were analysed parametrically using independent $t$ tests, whereas others were analysed non- parametrically (Mann-Whitney U test). Modal categories are reported as the measure of central tendency.

\section{RESULTS}

\section{Driving history questionnaire}

Drivers with PD and controls had similar driving experience (modal category for both groups, "4l-50 years"; $\mathrm{U}=238$; $\mathrm{p}=0.56)$. Similarly, there were no significant group differences for frequency of driving (modal category for both groups, "every day"; $\mathrm{U}=238$; $\mathrm{p}=0.55$ ), amount of time driven each week (modal category for participants with PD, "3-4 hours/week" and for the controls, "> 7 hours/week"; $\mathrm{U}=203.5 ; \mathrm{p}=0.18^{*}$ ), confidence felt when driving (modal category for both groups, "confident"; $U=236$; $p=0.51$ ), amount of long distance (modal category for both groups, "1-2 times/year"; $U=241.5 ; p=0.19$ ) or highway driving (modal category for both groups, "a few times each month"; $\mathrm{U}=215 ; \mathrm{p}=0.37$ ), or driving in unfamiliar areas (modal category for both groups, "confident"; $U=231$; $p=0.10$ ). There were also no significant differences between groups for self reported crashes (modal category for both groups, "no crash within past 10 years"; $U=259$; $p=0.92$ ), with seven of the 21 controls and eight of the 25 participants with PD reporting a crash within the previous 10 years.

However, the drivers with PD reported driving significantly fewer kilometres than controls (modal category, "60-90"

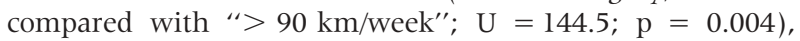
and were significantly less confident driving alone (modal category, "confident" compared with "very confident"; $\mathrm{U}=167.5 ; \mathrm{p}=0.015)$. Only three of the 36 driving conditions or tasks were rated as significantly more difficult by the drivers with PD (on a scale of 1 to 5 ): reading road

*This apparent difference was caused by a non-normal distribution: a subgroup of the controls drove longer distances. However, the mean values were in the same category of $3-4$ hours/week.

Table 3 Locations at which driving errors were made by the two groups as assessed by the occupational therapist

\begin{tabular}{|c|c|c|c|c|}
\hline \multirow[b]{2}{*}{ Locations } & \multicolumn{2}{|c|}{ Participant groups } & \multicolumn{2}{|l|}{ Statistics } \\
\hline & Controls & Parkinson's & t Value & p Value \\
\hline Roundabouts & $4.71(0.68)$ & $4.56(0.75)$ & 0.15 & 0.88 \\
\hline Merging & $1.33(0.26)$ & $2.16(0.37)$ & -1.75 & 0.09 \\
\hline Pulling in/pulling out & $4.19(0.36)$ & $3.92(0.29)$ & 0.59 & 0.56 \\
\hline Traffic light controlled intersection & $6.23(1.00)$ & $10.36(1.73)$ & -1.95 & 0.05 \\
\hline Non-traffic light controlled intersections, stop and give way & $4.19(0.86)$ & $6.64(1.17)$ & -1.64 & 0.11 \\
\hline Reversing, car parking & $2.28(0.57)$ & $3.84(0.54)$ & -1.98 & 0.05 \\
\hline One way (no oncoming traffic) & $3.33(0.67)$ & $4.92(0.70)$ & -1.62 & 0.11 \\
\hline Two way (oncoming traffic) & $6.09(1.38)$ & $7.72(1.11)$ & -0.92 & 0.36 \\
\hline Lane changing & $5.85(1.02)$ & $8.84(1.11)$ & -1.94 & 0.05 \\
\hline
\end{tabular}


signs in daylight $\left(\mathrm{t}_{44}=2.41 ; \mathrm{p}=0.02\right)$, moving their foot from one pedal to another $\left(\mathrm{t}_{44}=2.73 ; \mathrm{p}=0.009\right)$, and steering $\left(\mathrm{t}_{44}=2.80 ; \mathrm{p}=0.008\right)$. However, in all cases, groups differed by no more than one category-for example, "fairly easy" versus "quite easy".

\section{Driver safety ratings: occupational therapist and driving instructor assessments}

The safety ratings recorded for each of the participants with $\mathrm{PD}$ and the controls by the occupational therapist and driving instructor were highly correlated (fig 1), so we used the mean of the two raters' scores. These show that the drivers with PD were rated as significantly less safe on a scale of 1-10 (mean, 4.80; SD, 1.91) than the controls (mean, 6.56; SD, 1.72) $\left(\mathrm{t}_{44}=3.26 ; \mathrm{p}=0.002\right)$.

When the safety ratings were considered individually, five of the 25 drivers with PD scored between 1 and 3, a score defined as a driver for whom the instructor had to take action to avoid an incident, or the driver hit a significant object and should consider ceasing driving. Furthermore, 14 of the 25 drivers with PD scored 5 or less, indicating that they would have failed the Queensland driving test, as compared with five of the 21 controls.

Examination of the safety ratings of the drivers with PD and clinical disease indicators showed that time since diagnosis was significantly correlated with driver safety ratings $(r=-0.60 ; \mathrm{p}=0.001)$ - the longer the time since diagnosis, the lower the patient's rating (fig 2). This association remained significant even when age was partialled out $\left(r_{1,2.3}=-0.47 ; \mathrm{p}=0.02\right)$. However, driver safety ratings were not significantly correlated with either on time UPDRS $(r=-0.24 ; \mathrm{p}=0.25)$, the Hoehn and Yahr scale $(r=-0.06 ; \mathrm{p}=0.79)$, or levodopa dosage $(r=-0.36 ; \mathrm{p}=0.11)$.

\section{Self reported driving and safety rating}

Those in the PD group rated their driving skills more poorly compared with an average driver $(M=2.98)$ than did the controls $(M=2.57)$, and these differences reached significance $\left(\mathrm{t}_{44}=-2.7 \mathrm{l} ; \mathrm{p}=0.009\right)$. However, those with PD did not rate the driving route and conditions as harder than the control participants (patients with $\mathrm{PD}, \mathrm{M}=3.4$; controls, $\left.\mathrm{M}=3.19 ; \mathrm{t}_{44}=-1.20 ; \mathrm{p}=0.24\right)$. Importantly, there was no relation between an individual's self rating of driving performance and their safety rating $(r=-0.22$; $\mathrm{p}=0.14)$, and this was the case for both the drivers with PD and the controls.

\section{Types and locations of driving errors}

The overall safety scores recorded by the occupational therapist were significantly worse for the PD group than for controls, and both groups made proportionally more errors under self directed than directed navigation, but these differences did not reach significance (table 2). The drivers with PD made significantly more errors involving lane keeping and observation or monitoring of the blind spot than did the controls.
Table 3 shows the breakdown of errors by location type. The drivers with PD made significantly more errors at traffic light controlled intersections and at locations involving reversing, car parking, and lane changing.

\section{Driving instructor}

Apart from rating the drivers with PD as less safe than controls, the driving instructor also had to intervene more often to avoid an incident than for the controls (table 4). The drivers with PD also made significantly more critical errors than did the controls.

\section{DISCUSSION}

The drivers with PD were rated as significantly less safe than control participants by both the occupational therapist and the driving instructor on a standardised open road course. This finding is in general agreement with that of Heikkilä et al, ${ }^{14}$ who reported that $35 \%$ of drivers with PD were rated as unsafe to drive compared with control subjects. Radford et al reported similar findings but did not include a control group. ${ }^{15}$

The drivers with PD made significantly more errors than controls in lane keeping and lane changing, reversing, parking, monitoring of their blind spot, and negotiating traffic light controlled intersections. The driving instructor also had to intervene significantly more often for drivers with PD. These errors probably stem from some combination of decreased motor control skills, impaired visuo-spatial processing, sequence control, and planning in the drivers with PD. However, the current data do not allow this impaired performance to be attributed to any specific process. These practical skill findings agree with the questionnaire data from the drivers with PD, which showed that these patients had significantly more problems with steering and moving their feet between pedals. They also confirm the findings of Radford et $a l^{15}$ that patients with PD display a lack of observation at junctions, poor road positioning, and poor driving at roundabouts. However, their results must be considered with caution because of their limited sample size.

Importantly, the global driver safety ratings could not be predicted by the on time UPDRS or the Hoehn and Yahr scale, but had a stronger association with disease duration. Although previous studies have reported that various indices of driving performance have been predicted by the Webster's, ${ }^{8}$ Hoehn and Yahr, ${ }^{12}$ and UPDRS scales, ${ }^{10}$ there has been considerable disagreement in the literature. "Some researchers have used driving simulators, which have been shown to be poor predictors of true performance, ${ }^{11}$ or report on small and potentially unrepresentative samples. Indeed, Heikkilä et al found that speed of visual processing, medication use, and age were better predictors of open road driving performance than were clinical disease indicators, although, as in our study, the association between levodopa dosage and driving safety alone was not strong. ${ }^{14}$ These findings confirm the fact that currently used clinical disease markers do not adequately capture those aspects of PD that are linked with unsafe driving performance.

Table 4 Driving performance scores recorded by the driving instructor

\begin{tabular}{|c|c|c|c|c|}
\hline \multirow[b]{2}{*}{ Driving scores } & \multicolumn{2}{|c|}{ Participant groups } & \multicolumn{2}{|l|}{ Statistics } \\
\hline & Controls & Parkinson's & t Value & p Value \\
\hline Instructor interventions & $0.14(0.08)$ & $0.64(0.18)$ & -2.36 & 0.02 \\
\hline Critical errors & $0.76(0.30)$ & $2.24(0.58)$ & -2.14 & 0.04 \\
\hline Non-critical errors & $3.09(0.32)$ & $3.44(0.37)$ & -0.69 & 0.49 \\
\hline
\end{tabular}


The questionnaire findings suggest that the drivers with PD in this volunteer sample limit their own driving habits only to a minor degree, driving as often as controls, but covering fewer kilometres and driving alone less often. These findings are not surprising given that the individuals with PD and controls reported similar driving confidence levels. Importantly, an individual's self rated performance was a poor predictor of their driving safety, and this was true for all participants. This finding confirms the conclusion of Heikkilä et al, ${ }^{14}$ and suggests that it is unrealistic to expect individuals, with or without PD, to determine their own safety levels. Instead, it emphasises the importance of identifying objective, evidence based predictors of driving safety, which can be used to inform the treating practitioner and the licensing authority, and to educate and assist drivers in the use of compensatory behaviours. This was the objective of a larger study conducted by our research group, which includes the evaluation of visual, cognitive, and motor tests as predictors of safe driving performance.

It is also important to note that the drivers with PD in our study had chosen to continue driving, had retained their licence, and had volunteered to participate in our study-and are therefore likely to drive better than average, given that many patients with PD choose to stop driving..$^{20}$ The current results probably overestimate the driving performance of the wider PD population, and may under-represent the true driving performance decrement that accompanies the disease. In addition, these driving performance scores were obtained during optimal on time medication and do not address the impact of symptom fluctuations in PD.

\section{CLINICAL IMPLICATIONS}

Together, these findings have important implications that should be considered by health professionals dealing with patients with PD.

- At diagnosis, drivers with PD need advice regarding the potential impact of PD on driving, given that our study provides clear evidence that drivers with PD are significantly less safe than control drivers.

- Drivers with PD probably have limited awareness of their deteriorating driving performance and need regular monitoring. It is unrealistic to expect individuals, particularly those with $\mathrm{PD}$, to be aware that their driving is becoming unsafe and to adopt compensatory behaviours. This clearly indicates the importance of identifying predictors of unsafe performance and making them available for use by a patient's neurologist and to assist in the licensing process.

- Appropriately timed referral to occupational therapy driving assessment services to determine fitness to continue driving or the need for driving rehabilitation may enable drivers with PD to make more realistic plans for the future, whether to limit their driving, or stop altogether.

- Drivers with PD may benefit from targeted driver retraining. Our study clearly indicates that there are specific areas of difficulty that are more common in individuals with PD. It may be possible to use targeted interventions and retraining to prolong the length of time that patients with PD might drive safely and hence maintain their independence.

\section{ACKNOWLEDGEMENTS}

We thank J Wilson for patient management and her role in testing, I Booker for assistance in collection of the open road driving assessment data, and those who volunteered their time to participate in the study. Parkinson's Queensland Inc are thanked for their assistance with patient recruitment; J Rawlins is thanked for her particular involvement in this role. This study was supported by the Centre for Accident and Road Safety Research, Queensland (CARRSQ 99039).

\section{Authors' affiliations}

J M Wood, K Mallon, School of Optometry, Queensland University of Technology, Victoria Park Road, Kelvin Grove Q4059, Australia C Worringham, G Kerr, School of Human Movement Studies, Queensland University of Technology

P Silburn, Neurology Department, PA Hospital, Brisbane, Q4102 Australia

Competing interests: none declared

\section{REFERENCES}

1 Comella CL. Daytime sleepiness, agonist therapy, and driving in Parkinson's disease. JAMA 2002;287:509-11.

2 Homann CN, Wenzel K, Suppan K, et al. Sleep attacks in patients taking dopamine agonists: a review. BMJ 2002;324:1483-6.

3 Homann CN, Suppan K, Homann B, et al. Driving in Parkinson's disease-a health hazard? J Neurol 2003;250:1439-46.

4 Low KA, Miller J, Vierck E. Response slowing in Parkinson's disease: a psychophysiological analysis of premotor and motor processes. Brain 2002; 125:1980-94

5 Smiley-Oyen AL, Worringham CJ, Cross CL. Practice effects in threedimensional sequential rapid aiming in Parkinson's disease. Mov Disord 2002; 17:1196-204

6 Serrien DJ, Steyvers M, Debaere F, et al. Bimanual coordination and limbspecific parameterization in patients with Parkinson's disease. Neuropsychologia 2000;38:1714-22.

7 Lieb K, Brucker S, Bach M, et al. Impairment in preattentive visual processing in patients with Parkinson's disease. Brain 1999;122:303-13.

8 Madeley P, Hulley JL, Wildgust $H$, et al. Parkinson's disease and driving ability. J Neurol Neurosurg Psychiatry 1990;53:580-2.

9 Lings S, Dupont E. Driving with Parkinson's disease: a controlled laboratory investigation. Acta Neurol Scand 1992;86:33-9.

10 Zesiewicz TA, Cimino CR, Malek AR, et al. Driving safety in Parkinson's disease. Neurology 2002;59:1787-8.

11 Hakamies-Blomqvist L, Östlund J, Henriksson P, et al. Elderly car drivers in a simulator-a validation study. Linköping, Sweden: Swedish National Road and Transport Research Institute (VTI) SE-581 95, 2004

12 Dubinsky RM, Gray C, Husted D, et al. Driving in Parkinsons's disease. Neurology 1991;41:517-20.

13 McGwin G, Owsley C, Ball K. Identifying crash involvement among older drivers: agreement between self-report and state records. Accid Anal Prev 1998:30:781-91.

14 Heikkilä VM, Turkka J, Korpelainen J, et al. Decreased driving ability in people with Parkinson's disease. J Neurol Neurosurg Psychiatry 1998:64:325-30

15 Radford KA, Lincoln NB, Lennox G. The effects of cognitive abilities on driving in people with Parkinson's disease. Disabil Rehabil 2004; 26:65-70.

16 Heun R, Papassotiropoulos A, Jennssen F. The validity of psychometric instruments for detection of dementia in the elderly general population. Int J Geriatr Psychiatry 1998;13:368-80.

17 Wood JM. Age and visual impairment decrease driving performance as measured on a closed-road circuit. Hum Factors 2002:44:482-94.

18 Wood JM, Mallon K. Comparison of driving performance of young and old drivers (with and without visual impairment) measured under in-traffic conditions. Optom Vis Sci 2001;78:343-9.

19 Mallon K, Wood JM. Quantitative assessment of open road driving performance: effects of aging and early visual impairment. Am J Occup Ther 2004:58

20 Campbell MK, Bush TL, Hale WE. Medical conditions associated with driving cessation in community-dwelling, ambulatory elders. J Gerontol 1993;48:S230-4 


\section{PostScript}

\section{LETTERS}

\section{Possible transcallosal seizure induction by paired pulse transcranial magnetic stimulation in a patient with frontal lobe epilepsy} cranial magnetic stimulation (TMS) has been reported in normal subjects and by single pulse TMS close to the epileptic focus in patients with epilepsy.

\section{Case report}

We report an 18 year old patient with right frontal lobe epilepsy due to paramedian focal cortical dysplasia (FCD). The patient's usual seizure semiology consisted of a somatosensory aura of the left hand followed by a tonic seizure of the left arm which evolved to a bilateral asymmetrical tonic seizure without loss of consciousness. In the two years preceding the study (see below) he had rare night-time seizures only. His antiepileptic medication consisted of levetiracetam $500 \mathrm{mg}$, phenobarbital $25 \mathrm{mg}$, and carbamazepine $1600 \mathrm{mg}$ daily.

During presurgical videoelectroencephalogram (video-EEG) monitoring, interictal EEG showed right frontotemporal spikes. Ictal EEG revealed seizure patterns with a right frontal onset. Magnetic resonance imaging (MRI) showed FCD in the right superior frontal gyrus extending into the right precentral gyrus (fig 1A). Neurological examination was normal.

\section{Transcranial magnetic stimulation} a protocol described previously ${ }^{2}$ to evaluate
Seizure induction by high frequency trans-

The patient participated in a TMS study using

intracortical excitability of both motor cortices (Ml). The study was approved by the local ethics committee, and the patient gave written informed consent.

We used a focal $70 \mathrm{~mm}$ figure of eight coil connected to two magnetic stimulators via a BiStim module (Magstim Company, Dyfed, UK). Surface electromyography (EMG) was recorded from the contralateral abductor digiti minimi muscle (ADM) of the hand.

TMS commenced over the left Ml contralateral to the epileptic focus with the coil placed over the Ml hand area. First, motor thresholds (RMT, AMT) and cortical induced silent period at an intensity of $110 \%$ RMT were evaluated. Next, paired pulse TMS (conditioning stimulus set at $38 \%$ of maximum stimulator output, second stimulus $60 \%$ of stimulator output) was started on the left Ml with a train of paired pulses with ISI $2,3,10$, and $15 \mathrm{~ms}$ in a random order.

After 65 stimuli, the patient noticed that his habitual somatosensory aura of the left hand followed by myoclonic jerks of the left forearm (mainly biceps brachii muscle and forearm flexor muscles) was triggered by each stimulus, contralateral to the epileptogenic zone but ipsilateral to the cortical stimulation. The jerks were triggered by both single and paired stimuli at all ISI and rapidly involved both arms. These motor phenomena were different from the typical seizure semiology. EMG recordings of the ADM showed movement artefacts 63-75 ms after the MEP (fig 1B). The TMS was immediately interrupted, which aborted the myoclonus at once.

The TMS data of the left hemisphere showed increased motor thresholds, prolonged cortical induced silent period, markedly decreased intracortical inhibition, and increased facilitation compared with 20 controls $^{2}$ (percentiles of the patient's measures within the control group: $>99 \%$ for ISI 2 and
A

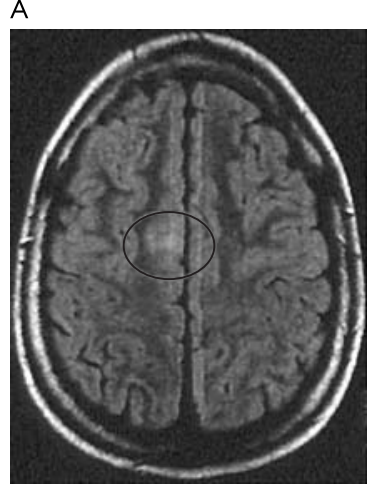

B

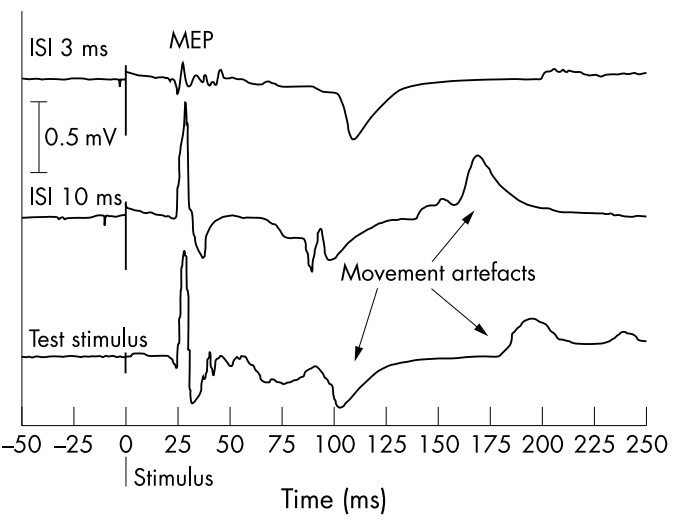

Figure 1 (A) Axial T2-weighted magnetic resonance imaging (MRI) scan (fluid attenuated inversion recovery (FLAIR)) of the patient's brain. T2 prolongation and blurring of grey-white junction represents focal cortical dysplasia of $1 \times 1 \mathrm{~cm}$ (see circle) located in the right superior frontal sulcus (extending to the right motor cortex, seen in further MRI slices). (B) Examples of motor evoked potentials (MEPs) from the right abductor digiti minimi muscle (ADM) during transcranial magnetic stimulation of the left hemisphere at different interstimulus intervals (ISI) and after unconditioned test stimulus. The patient showed stimulus triggered myoclonic jerks of both hands and forearms leading to movement artefacts in the ADM recordings.
CSP, $>95 \%$ for motor thresholds and ISI 15 , $>90 \%$ for ISI 10 , and $>85 \%$ for ISI 3 ).

After the TMS experiment, the patient was again free of daytime seizures until the last follow up visit six months later.

\section{Transcallosal seizure induction by paired pulse TMS}

In patients with epilepsy, all reported cases of seizure induction by TMS have occurred during ipsilateral stimulation and near to the epileptic focus. Therefore, it has been assumed that direct stimulation of the epileptogenic tissue was required to trigger a seizure. ${ }^{1}$ We used a focal coil placed over the left $\mathrm{Ml}$ hand area more than $5 \mathrm{~cm}$ away from the midline. Thus, it is unlikely that the right epileptogenic zone or mesial frontal cortex of the present patient was stimulated directly, and we assume that an indirect transcallosal activation of the epileptogenic zone provoked the aura. The latency of $65-75 \mathrm{~ms}$ of the myoclonic jerks after the MEP may reflect polysynaptic pathways in addition to a direct transcallosal connection of both $\mathrm{Ml}$. It is still not clear whether involvement of additional cortical areas such as the ipsilesional and contralesional sensory cortices or basal ganglia contributed to the seizure provocation. Despite the patient's statement that the jerks were not volitional, this cannot be completely ruled out. The preceding somatosensory aura, however, represented his typical seizure semiology. We hypothesise that transcallosal activation of the epileptic focus was promoted by the increased excitability of Ml, which was due to the underlying FCD. This, in turn, led to the aura and peri-ictal changes in Ml excitability facilitating TMS driven myoclonic jerking. It has been previously reported that FCD is intrinsically epileptogenic and promotes reflex seizures.

There is a possibility that ipsilateral pathways of movement activation could underlie our observations. In a child with extensive cortical dysplasia, TMS of the unaffected hemisphere evoked MEPs in both ADM muscles implying bilateral corticospinal connections from one cortex. ${ }^{4}$ Histological studies on severe brain damage in early development have revealed collateral sprouting into denervated areas of cortex or spinal cord. ${ }^{4}$ Ipsilateral activation under maximum muscle contraction has been observed in healthy volunteers and in patients with acute stroke. ${ }^{5}$ Our patient, however, presumably had congenital but circumscribed FCD, no motor deficits, and was investigated at rest. This and the fact that his habitual somatosensory aura occurred before the myoclonic jerks strongly argue against the activation of ipsilateral corticospinal tracts. Activation of a silent mirror focus in the left hemisphere with subsequent spread to the right is also unlikely because exclusively right sided ictal and interictal epileptiform discharges were recorded during the video-EEG monitoring.

\section{Changes in motor cortex excitability} Our patient's higher motor thresholds compared with controls are very likely due to his ion channel blocking anticonvulsant medication. ${ }^{6}$ 
The loss of intracortical inhibition and increased intracortical facilitation in the left hemisphere contralateral to the epileptogenic zone may reflect synaptic reorganisation of the ipsilesional and contralesional motor cortices. These distant functional cortical changes associated with malformations of cortical development have also been described previously. ${ }^{7}$ The prolongation of the cortical induced silent period seen in the present patient may be independent of the phenobarbital intake ${ }^{6}$ and confirms similar findings from previous studies as a remote effect of FCD on the motor cortex in untreated patients with cortical dysgenesis.

\section{Conclusion}

Unilateral epileptogenic FCD involving $\mathrm{Ml}$ can induce complex bilateral alteration of motor cortex excitability resulting in a net increase of excitability. In such cases, transcallosal seizure induction appears to be possible with paired pulse TMS using a focal coil away from the epileptic focus.

J Reis, F Rosenow, B Fritsch, S Knake, W H Oertel, H M Hamer

Interdisciplinary Epilepsy-Center, Department of Neurology, Philipps-University Marburg, Germany

Correspondence to: J Reis, Interdisciplinary EpilepsyCenter, Department of Neurology, Philipps-University Marburg, Rudolf-Bultmann-Str. 8, 35033 Marburg, Germany; reis@staff.uni-marburg.de

doi: 10.1136/jnnp.2004.042127

Competing interests: none declared

\section{Reference}

1 Classen J, Witte OW, Schlaug G, et al. Epileptic seizures triggered directly by focal transcranial magnetic stimulation. Electroencephalogr Clin Neurophysiol 1995;94:19-25.

2 Reis J, Tergau F, Hamer HM, et al. Topiramate selectively increases motor cortex excitability in human motor cortex. Epilepsia 2002;43: 1149-56.

3 Palmini A, Gambardella A, Andermann F, et al. Intrinsic epileptogenicity of human dysplastic cortex as suggested by corticography and surgical results. Ann Neurol 1995;37:476-87.

4 Maegaki Y, Yamamoto T, Takeshita K. Plasticity of central motor and sensory pathways in a case of unilateral extensive cortical dysplasia: investigation of magnetic resonance imaging transcranial magnetic stimulation, and shortlatency somatosensory evoked potentials. Neurology 1995;45:2255-61.

5 Caramia MD, Palmieri MG, Giacomini P, et al. Ipsilateral activation of the unaffected motor cortex in patients with hemiparetic stroke. Clin Neurophysiol 2000;111:1990-6.

6 Ziemann U, Lonnecker S, Steinhoff BJ, et al. Effects of antiepileptic drugs on motor cortex excitability in humans: a transcranial magnetic stimulation study. Ann Neurol 1996;40:367-78.

7 Cincotta M, Borgheresi A, Guidi L, et al. Remote effects of cortical dysgenesis on the primary motor cortex: evidence from the silent period following transcranial magnetic stimulation. Clin Neurophysiol 2000;1 11:1340-5

\section{Hashimoto's encephalopathy: steroid resistance and response to intravenous immunoglobulins}

Hashimoto's encephalopathy is a steroid responsive disorder characterised by high titres of anti-thyroid antibodies and manifesting as sub-acute onset of confusion, episodes of myoclonus, seizures, and strokelike episodes. Although excellent response to steroids is characteristic, other treatments such as plasmapheresis or administration of azathioprine or cyclophosphamide have been occasionally tried. We report a case of initially steroid responsive Hashimoto's encephalopathy which became steroid resistant and then responded well to intravenous immunoglobulins.

\section{Case report}

A 29 year old woman was admitted in 1987 with an episode of headache, confusion, agitation, and hallucination. She had a mild fever and was thought to have neck stiffness. A CT scan was normal as were the inflammatory markers. CSF examination showed 9240 red cells and 33 white cells (45\% polymorphs and 55\% lymphocytes). CSF protein, glucose, and microbiology were normal. A presumed diagnosis of meningoencephalitis was made and the patient was treated with acyclovir and antibiotics. The patient made a good recovery but was readmitted a week later with agitation and confusion with pain and weakness down the left side. No focal neurology was found on examination and the patient was thought to be suffering from an anxiety state.

During the next 14 years, the patient was admitted on several occasions with episodes of confusion and agitation: investigations including lumbar puncture, CT scans, EEG, thyroid function, porphyria screens, autoantibody screens (including antinuclear antibodies, ANCA, and those against extractable nuclear antigens), and metabolic and septic screens were found to be normal. She had been admitted to the psychiatry unit and was thought to be suffering from acute mania or a dissociative state, precipitated by stress and sleep deprivation. In 2001, she was referred to the neurology clinic for similar episodes, which were increasing in frequency.

On first review in the neurology clinic, clinical examination was unremarkable except for bilaterally symmetrical and brisk reflexes. MRI scan of the brain and EEG were repeated and found to be normal. Thyroid peroxidase antibody was raised at $250 \mathrm{IU} / \mathrm{ml}$ (normal range 0-60). Thyroid function tests were normal.

She remained well until April 2003 when a further episode of drowsiness and confusion occurred (thyroid peroxidase antibody $266 \mathrm{IU} / \mathrm{ml}$ ). This responded remarkably and within $24 \mathrm{~h}$ to a course of dexamethasone given intravenously at a dose of $16 \mathrm{mg}$ daily for 5 days. An EEG repeated during the episode showed diffuse slowing but no epileptiform abnormalities.

The patient was re-admitted in May 2004 with another episode of confusion and agitation. Interestingly, this episode occurred after a 5 day course of oral prednisolone $60 \mathrm{mg} /$ day for a chest infection. Apart from the acute confusional state there was no focal neurology. Repeat CT scan and CSF examination including oligoclonal bands were within normal limits. Repeat thyroid peroxidase antibody was elevated at $272 \mathrm{IU} / \mathrm{ml}$ (normal range $0-60$ ). Voltage gated potassium channel antibodies were negative. Other investigations including thyroid function remained normal. EEG showed diffuse bilateral slow wave activity. A 7 day course of intravenous dexamethasone $16 \mathrm{mg} /$ day was initiated. The confusion and agitation worsened and later she became very drowsy. Intravenous immunoglobulin ( $400 \mathrm{mg} / \mathrm{kg}$ daily) was given causing a dramatic improvement within $12 \mathrm{~h}$. Unfortunately, on the same day, the patient developed an increase in alanine transaminase and C-reactive protein levels. A possible reaction to intravenous immunoglobulin was considered and the infusion was discontinued. Later, the increased levels were confirmed to be secondary to septicaemia from a Staphylococcus aureus infected cannula site. The inflammatory markers normalised after a course of antibiotics. During this time the patient remained drowsy, confused, and occasionally agitated. A 5 day course of intravenous immunoglobulin was reinstated. Again, there was a dramatic improvement within $24 \mathrm{~h}$ and she was discharged home at the end of the course having completely recovered.

\section{Discussion}

Hashimoto's encephalopathy (HE) is a steroid responsive disorder characterised by high titres of anti-thyroid antibodies. The original description of this condition was in an established case of Hashimoto's thyroiditis where the patient developed focal neurological deficits and coma. ${ }^{1}$ Clinical presentation includes episodic confusion, myoclonus, seizures, and stroke-like episodes. ${ }^{2}$ Females are more affected than males (3.6:1), with a mean age of onset of 41 years. The hallmark of $\mathrm{HE}$ is its response to steroids, most cases improving within a few hours to days. ${ }^{3}$ The titres of anti-thyroid antibodies maybe independent of the severity of the clinical presentation. ${ }^{4}$ Fewer than 100 cases of HE have been reported in the literature. Goitre and hypothyroidism can be associated with the disorder, but the majority of patients are euthyroid. Although steroid responsiveness is the rule, additional immunosuppressive therapy in the form of azathioprine and cyclophosphamide has been tried in a minority of patients. ${ }^{23}$ One case report of HE responding to plasmapheresis is documented. ${ }^{5}$ There was no benefit in the two documented cases where intravenous immunoglobulin was administered. $^{56}$

Several pathophysiological hypotheses have been suggested for HE. The initial report of HE suggested a vascular aetiology followed by localised cerebral oedema as a possible mechanism. ${ }^{1}$ Some authors suggest that the CSF thyroid autoantibodies may react with a putative CNS antigen and form immune complexes. $^{24}$ The immunopathological basis of this syndrome has been compared to a relapsing form of acute disseminated encephalomyelitis. ${ }^{7}$ Although reversible MRI findings have been described in $\mathrm{HE}^{8}$ neuroimaging (except for isolated patchy uptake by isotope scans) is usually normal in most cases. ${ }^{3}$ Cerebral angiography has been found to be normal in several cases of HE, unlike in many other cerebral vasculitides. ${ }^{1-3} 5$

Thyroid autoantibodies can co-exist with several other forms of autoimmune encephalomenigitis, but the normal MRI scan, the initial dramatic response to steroids, and negative autoantibodies for most other common vasculitides, tends to favour the diagnosis of $\mathrm{HE}$ in our case. Steroid responsive encephalopathy associated with Hashimoto's thyroiditis is an alternately proposed name for this condition, ${ }^{9}$ but the vast majority of cases have normal thyroid function, leaving "Hashimoto's encephalopathy" a universally accepted term. A recent literature review of 85 patients with encephalopathy and antithyroid antibodies suggests that the combination of encephalopathy, high serum anti-thyroid antibody concentrations, and 
responsiveness to glucocorticoid therapy seems unlikely to be due to chance. ${ }^{10}$

The initial meningo-encephalitic type presentation of our patient in 1987 was probably the first manifestation of $\mathrm{HE}$ in view of clinical findings and laboratory data (Mild CSF pleocytosis is not unusual in HE. ${ }^{3}$ ) There was a delay of 14 years before the diagnosis was first established, in spite of several hospital admissions. The initial relapses after diagnosis responded well to steroids, confirming the diagnosis of HE. Whether the current episode was precipitated by the sudden withdrawal of oral steroids or the chest infection itself, for which they were prescribed, is unclear.

Our patient illustrates the possibility of steroid resistance in an established case of $\mathrm{HE}$ and the need to consider further immunomodulatory therapy. Intravenous immunoglobulins are a safe, convenient, and effective treatment in such circumstances.

S Jacob, Y A Rajabally Department of Neurology, Leicester Royal Infirmary, University Hospitals of Leicester, Leicester LE1 5WW

Correspondence to: $\operatorname{Dr}$ Y A Rajabally, Department of Neurology, University Hospitals of Leicester, Royal Infirmary, Leicester LE1 5WW, UK; yusuf.rajabally@ uhl-tr.nhs.uk

doi: 10.1136/jnnp.2004.049395

Competing interests: none declared

\section{References}

1 Lord Brain, Jellinek EH, Ball K. Hashimoto's disease and encephalopathy. Lancet 1966;2:512-4.

2 Shaw PJ, Walls TJ, Newman PK, et al. Hashimoto's encephalopathy: a steroidresponsive disorder associated with high antithyroid antibody titre - report of 5 cases. Neurology 1991;41:228-33.

3 Peschen-Rosin R, Schabet M, Dichgans J. Manifestation of Hashimoto's encephalopathy years before onset of thyroid disease. Eur Neurol 1999:41:79-84

4 Ferraci F, Moretto G, Candeago RM, et al. Antithyroid antibodies in the CSF. Their role in the pathogenesis of Hashimoto's encephalopathy. Neurology 2003;60:712-4.

5 Boers PM, Colebatch JG. Hashimoto's encephalopathy responding to plasmapheresis. J Neurol Neurosurg Psychiatry 2002;73:601.

6 Henchey R, Cibula J, Helveston W, et al. Electroencephalographic findings in Hashimoto's encephalopathy. Neurology 1995;45:977-81.

7 Chaudhuri A, Behan PO. The clinical spectrum, diagnosis, pathogenesis and treatment of Hashimoto's encephalopathy (recurrent acute disseminated encephalomyelitis). Curr Med Chem 2003; 10(19): 1945-53

8 Bohnen NI, Parnell KJ, Harper CM. Reversible MRI findings in a patient with Hashimoto's encephalopathy. Neurology 1997;49:246-7.

9 Mahmud FH, Renaud DL, Reed AM, et al. Steroidresistant encephalopathy associated with Hashimoto's thyroiditis in an adolescent with chronic hallucinations and depression: case report and review. Pediatrics 2003; 112(3 Pt 1):686-90.

10 Chong JY, Rowland LP, Utiger RD. Hashimoto's encephalopathy. Syndrome or myth? Arch Neurol 2003:60:164-71

\section{Spontaneous lobar haemorrhage in CADASIL}

CADASIL is an autosomal dominant form of arteriopathy, primarily affecting cerebral vessels, and predominantly caused by point mutations in the Notch 3 gene on the short arm of chromosome 19. ${ }^{1}$ Affected individuals develop subcortical strokes and cognitive deficits in their 50s and $60 \mathrm{s.}^{2}$ Brain magnetic resonance imaging (MRI) shows large areas of leukoencephalopathy and multiple subcortical lacunar infarcts. Small arteries and capillaries are characterised histologically by a non-atherosclerotic, non-amyloid angiopathy with accumulation of granular osmiophilic material (GOM) within the smooth muscle cell basement membranes and extracellular matrix. ${ }^{3}$ While CADASIL is considered a primarily ischaemic form of vascular dementia, microhaemorrhages have recently been reported in $31 \%$ of symptomatic Notch 3 mutation carriers, suggesting that structural fragility of the arterial walls may lead to leaking of haem products. ${ }^{4}$ Lobar haemorrhage in the absence of other risk factors for haemorrhage has previously been reported in one patient with CADASIL. ${ }^{5}$ Here we report a second case.

\section{Case report}

A 56 year old man who had been diagnosed with multiple sclerosis six years earlier was admitted to the hospital with an acute change in mental state. He had collapsed at home and was unresponsive when rescue arrived. In the emergency room he had a depressed level of consciousness and difficulty following commands, with paucity of speech, dysarthria, and hypophonia. There was no evidence of head trauma. His blood pressure was $100 / 63 \mathrm{~mm} \mathrm{Hg}$ and his temperature was $36.1{ }^{\circ} \mathrm{C}$

Past medical history included chronic obstructive pulmonary disease, prostate resection for prostate cancer, and a history of nicotine and alcohol dependence. He had no history of hypertension, diabetes mellitus, or coagulopathy. His drug treatment included ipratropium, ranitidine, methyprednisolone, and albuterol. His mother, now deceased, had been diagnosed as having multiple sclerosis and had migraines with auras, stroke-like symptoms, and dementia. He had eight siblings, three with headaches and one with recent transient ischaemic events.

Computed tomography (CT) of the head in the emergency department showed an area of high attenuation in the right frontal lobe consistent with an acute intraparenchymal haemorrhage (fig lA). There was no evidence of trauma on head CT. Gradient echo MRI sequences of the brain done on hospital day 2 showed a $2 \times 2.5 \mathrm{~cm}$ area of haemorrhage in
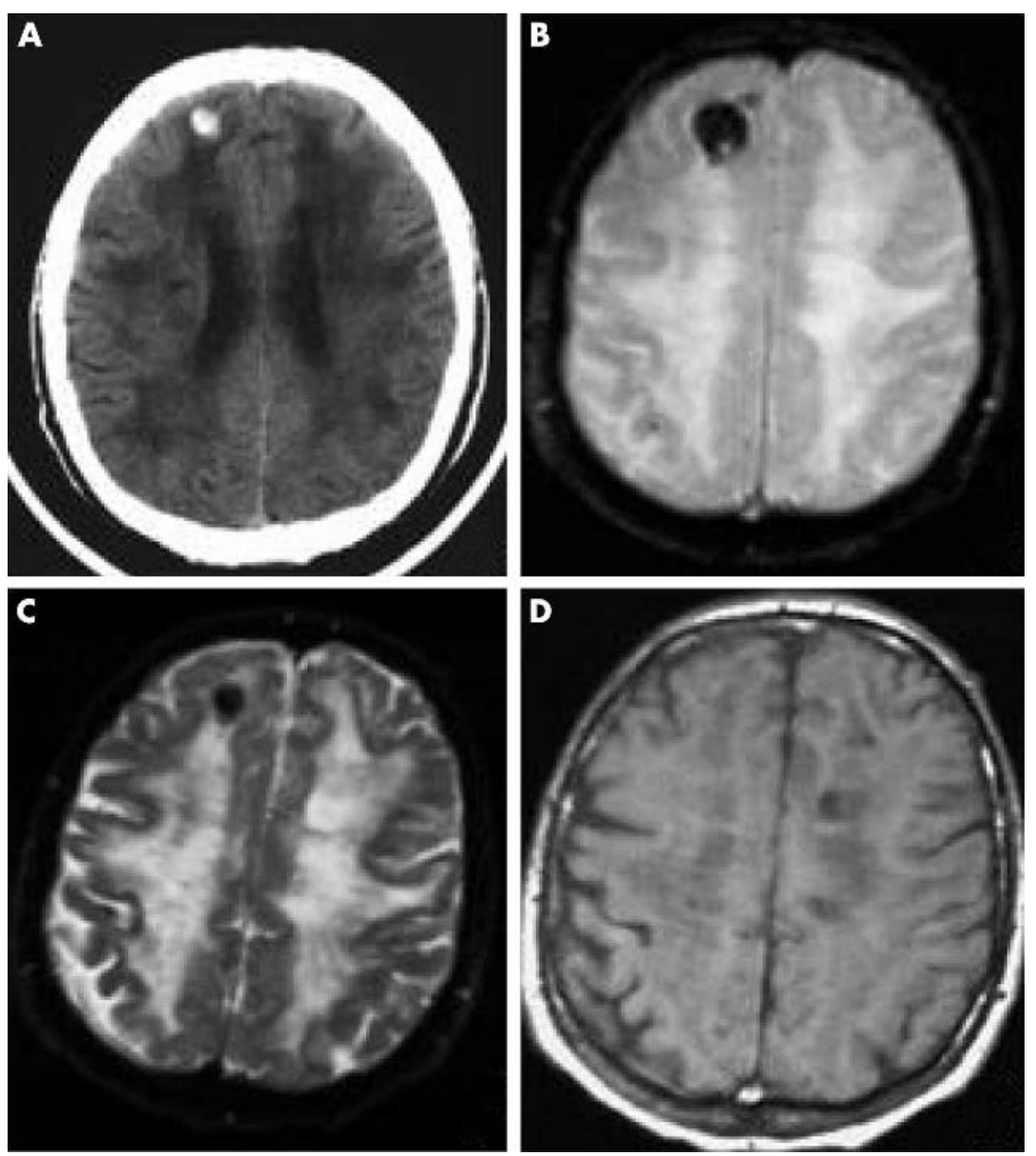

Figure 1 (A) Non-contrast computed tomography of the head done in the emergency room showing an area of high attenuation in the right frontal lobe consistent with acute haemorrhage. The other panels show non-contrast magnetic resonance imaging done on hospital day 2 : (B) Gradient echo sequence demonstrating a $2 \times 2.5 \mathrm{~cm}$ area of haemorrhage in right frontal lobe, a microhaemorrhage in the right parietal region, and extensive white matter disease. (C, D) The area of haemorrhage is hypointense on T2 and isointense on $\mathrm{T} 1$ weighted imaging, consistent with an acute haemorrhage. 
the superior-anterior aspect of the right frontal lobe white matter as well as a microhaemorrhage in the right parietal region (fig $1 \mathrm{~B}$ ). The area of haemorrhage was hypointense on T2 (fig IC) and isointense on $\mathrm{Tl}$ weighted sequences (fig ID), consistent with acute haemorrhage. There was no MRI evidence of a cavernous haemangioma, arteriovenous malformation, or tumour. Magnetic resonance angiography was not done.

A brain biopsy of the right frontal lobe done on the seventh hospital day showed degeneration of small and medium sized arteries. Vessel walls were thick and hyalinised in the grey matter, white matter, and meninges. PAS staining was positive and the muscular coat of the large vessels revealed degenerative changes. Electron microscopy showed the granular osmiophilic material characteristic of CADASIL. Notch 3 gene testing revealed a R133C mutation in exon 4, consistent with the diagnosis of CADASIL. The patient remained normotensive throughout his hospital stay. On the fifth hospital day he developed aspiration pneumonia requiring mechanical ventilation. He died eight days later as a result of this pneumonia.

\section{Comment}

This is the second report of spontaneous cerebral haemorrhage in a patient with CADASIL. In 1977, Sourander and Walinder reported a 29 year old man with hereditary multi-infarct dementia on anticoagulants, with a large haemorrhage in the right hemisphere. ${ }^{5}$ This family was thought to be one of the first with CADASIL; however, recent testing for Notch 3 mutations in the family has not confirmed that diagnosis. ${ }^{6}$ In 1992, Baudrimont et al reported a case of massive left cerebral haematoma involving the caudate nucleus, internal capsule, and thalamus in a 40 year old normotensive woman who was a member of a large CADASIL family. She had no known history of other risk factors for haemorrhage. ${ }^{7}$

The index patient in this report had no evidence of coagulopathy and no history of previous hypertension, cerebral haemorrhage, or anticoagulant therapy. The patient could have experienced a haemorrhagic contusion related to a closed head injury during his unwitnessed fall before admission, but there was no evidence of trauma on physical examination or on head CT. On MRI there was no evidence of a cavernous haemangioma, arteriovenous malformation, or neoplasm. Necropsy was not carried out.

Ultrastructural analysis of small arteries in human postmortem brain and skin in patients with CADASIL shows breakdown of the arterial wall cytoarchitecture, which may help explain the propensity for microhaemorrhages. ${ }^{8}$ The first notch 3 transgenic mouse shows early widening of the subendothelial and intra-smooth-muscle spaces in the vascular smooth muscle cells, denoting weakening of the arterial wall and increasing susceptibility to micro- and macrohaemorrhages. ${ }^{9}$

This case report supports the growing evidence for both ischaemia and haemorrhage in a variety of small artery diseases including amyloid angiopathy and CADASIL. ${ }^{10}{ }^{11}$ Clinicians may need to consider the possibility of haemorrhage when evaluating new events and deciding on treatment for stroke prevention in patients with CADASIL.
Acknowledgements

Supported by grants P20 RR015578 and K08MH001487.

A V Maclean

Brown Medical School, Providence, Rhode Island, USA

R Woods, L M Alderson, S P Salloway Department of Clinical Neurosciences Brown Medical School

S Correia

Department of Psychiatry and Human Behavior Brown Medical School

S Cortez, E G Stopa

Department of Pathology (Neuropathology Division) Brown Medical School

Correspondence to: Professor Stephen Salloway, Department of Neurology, Butler Hospital, 345 Blackstone Blvd, Providence, RI 02906, USA Stephen_Salloway@brown.edu

doi: 10.1136/jnnp.2004.042564

Competing interests: none declared

\section{References}

1 Joutel A, Corpechot C, Ducros A, et al. Notch3 mutations in CADASIL, a hereditary adult-onset condition causing stroke and dementia. Nature 1996;383:707-10.

2 Dichgans M, Mayer M, Uttner I, et al. The phenotypic spectrum of CADASIL: clinical findings in 102 cases. Annals of Neurology 1998;44:731-9.

3 Ruchoux MM, Maurage CA. CADASIL: cerebral autosomal dominant arteriopathy with subcortical infarcts and leukoencephalopathy. J Neuropathol Exp Neurol, 1997; 56:947-64.

4 Lesnik Oberstein SA, van den Boom R, van Buchem MA, et al. Cerebral microbleeds in CADASIL. Neurology 2001;57:1066-70.

5 Sourander P, Walinder J. Hereditary multi-infarct dementia: morphological and clinical studies of a new disease. Acta Neuropathol (Berlin) 1977:39:247-54.

6 Kalimo H, Ruchoux MM, Viitanen $M$, et al. CADASIL: a common form of hereditary arteriopathy causing brain infarcts and dementia. Brain Pathol 2002; 12:371-84.

7 Baudrimont M, Dubas F, Joutel A, et al. Autosomal dominant leukoencephalopathy and subcortical ischemic stroke: a clinicopathological study. Stroke 1993;24:122-5.

8 Rouchoux MM, Maurage CA. Endothelial changes in muscle and skin biopsies in patients with CADASIL. Neuropathol Appl Neurobiol 1998;24:60-5.

9 Ruchoux MM, Domenga $\mathrm{V}$, Brulin $\mathrm{P}$, et al. Transgenic mice expressing mutant notch 3 develop vascular alterations characteristic of cerebral autosomal dominant angiopathy with subcortical infarcts and leukoencephalopathy. Am J Pathol 2003;162:329-42.

10 Greenberg SM. Cerebral amyloid angiopathy and vessel dysfunction. Cerebrovasc Dis 2002; 13(suppl 2):42-7.

11 Dichgans M, Holtmannspotter M, Herzog J, et al. Cerebral microbleeds in CADASIL: a gradientecho magnetic resonance imaging and autopsy study. Stroke 2003;34:376-7

\section{Urinary retention caused by a small cortical infarction}

The cortical representation of micturition is speculated to reside in the medial frontal lobes. ${ }^{12}$ Lesion pathology, however, varies from acute stroke to a neoplasm, and there is not necessarily a small, distinct lesion. ${ }^{2}$ We report a case of urinary retention in which the main presenting symptom is thought to have been caused by a small cortical infarction.

\section{Case report}

One morning, a 66 year old, right handed man had difficulty urinating. He had no history of voiding difficulty, diabetes mellitus, injury to the lower urinary tract, or neurological disease. Digital rectal examination and ultrasonography of the prostate detected no enlargement. Urinalysis showed no haematuria or pyuria. He was not taking any medications that cause voiding dysfunction. There was no urinary incontinence, but he had difficulty in voiding even though he felt the bladder was full. At that time, he also had difficulty in lifting his left arm and leg and so was brought to our hospital. Neurological examination in the emergency room found no weakness, and he was sent home. Later, he experienced urinary retention and visited the emergency room again. His post void residual urine volume was $350 \mathrm{ml}$, and an urinary catheter was inserted. At that time the patient was alert, and his cranial nerves were intact. Limb muscle strength was normal. Sensory examination was unremarkable. Tendon reflexes were normal in all four limbs. Tandem gait and standing on one foot were difficult. He had normal bladder sensation but difficulty in urinating. Drip infusion pyelography revealed no abnormality in the upper urinary tract or the form of the bladder. Filling cystometry showed stable detrusor with normal bladder sensation, whereas acontractile detrusor was noted in the voiding phase. He could void only with strain, having a peak flow rate of $5.0 \mathrm{ml} / \mathrm{s}$ and a voided volume of $135 \mathrm{ml}$. Diffusion weighted MRI, performed on the day of onset, showed a small, distinct, high intensity signal, and $\mathrm{Tl}$ weighted imaging showed a low signal in the right caudal part of the anterior cingulate gyrus, indicative of an infarct in the acute stage (fig 1A and C). No definitive infarct was observed elsewhere. MR angiography showed no occlusion or stenosis of the intracranial vessels. An electrocardiogram was normal. Transthoracic echocardiograms showed no abnormal findings. The urinary catheter was withdrawn 3 days after admission, and he had no subsequent difficulty with urination. His gait returned to normal about the same time.

\section{Discussion}

In the acute stage of a cerebral vascular accident, the presenting symptom often is urinary retention due to detrusor areflexia, ${ }^{3}$ but patients who have this problem usually have a major stroke with severe neurological deficits.

To the best of our knowledge, this is the first report in the English literature of urinary retention, although temporary, caused by a small cortical infarct as shown by diffusion weighted MRI.

Various cortical areas are activated during voiding because a network of brain regions is necessary for voiding modulation. ${ }^{4}$ The locations of the primary cerebrum cortical areas for voiding and storage are speculated to be separate, the former being at the para-central lobule. ${ }^{2}$ A PET study found normal micturition to be associated with activation of the middle frontal gyrus, superior frontal gyrus, superior precentral gyrus, thalamus, and the caudal part of the anterior cingulate gyrus in the left hemisphere. ${ }^{5}$ Another recent PET study showed that increased brain activity related to increasing bladder volume was located in the bilaterally mid-cingulate cortex, while that related to decreased urge to 

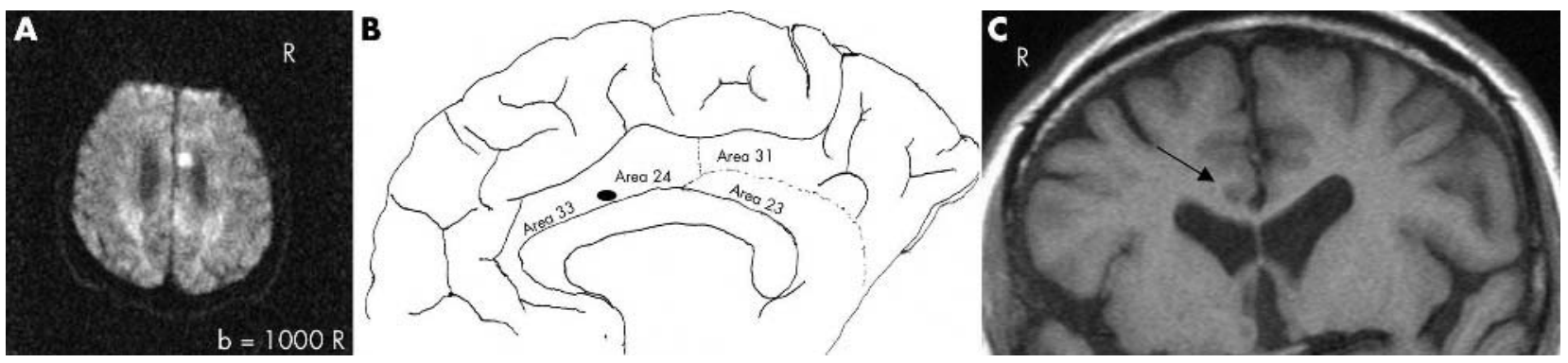

Figure 1 Diffusion weighted MRI. (A) The axial section shows a small high signal in the right caudal part of the anterior cingulate gyrus. (B) The closed ellipse denotes the lesion in the cortical map (map modified from Brodmann, 1909). (C) In the T1 weighted MRI procedure, the coronal section shows a low intensity signal in the right caudal part of anterior cingulate gyrus (arrow). (Imaging condition: a negative tilt of $-20^{\circ}$ to the orbitomeatal line.)

void was bilaterally in a different portion of the mid-cingulate gyrus. ${ }^{4}$

Although the infarct in our patient was located in the caudal part of the anterior cingulate gyrus, it was on the right side, nearby the region activated in the PET study. SPECT showed increased blood flow in the right medial frontal area, indicative that urinary retention was due to "decreased urge to void", and decreased flow in the right medial parietal lobe, which might explain the gait disturbance, in light of the essentially normal sensory examination. Unfortunately, a PET scan was not available in our hospital (Kameda Medical Center). Because there has been no report of an isolated lesion of the cingulate gyrus causing hemiparesis, these brain imaging studies indicate that the left hemiparesis, which disappeared within a half day of onset, could have been due to a transient ischaemic attack.

Urinary symptoms disappeared 3 days after admission, probably because the cortical neuron network compensated by providing a functional alternative to the lesion damaged by the infarct. This is similar to the condition of urinary incontinence after cerebral infarction, as is well documented. The laterality of the lesion in this patient differs from that in a previous PET study ${ }^{4}$ which showed bilateral activation in the cingulate gyrus. Because this report cites only a single case, its applicability is limited. Additional lesion studies of patients with micturition disturbance due to small cortical infarcts should help to identify the anatomical cerebral structures involved in voiding.

K Funakoshi, T Fukutake, H Nishino, S Sato Department of Neurology, Kameda Medical Center, Chiba, Japan

T Yamanishi

Department of Urology, Dokkyo University School of Medicine, Tochigi, Japan

Correspondence to: Dr Funakoshi, Department of Neurology, Dokkyo University School of Medicine, Kitakobayashi 880, Mibu, Shimotsuga, Tochigi 3210293, Japan; kei-f@dokkyomed.ac.jp

doi: 10.1136/jnnp.2004.050542

Competing interests: none declared

\section{References}

1 Sakakibara R, Hattori T, Yasuda K, et al. Micturitional disturbance after acute stroke: analysis of the lesion site by CT and MRI. J Neurol Sci 1996; 137:47-56.

2 Kuroiwa Y. Neurological bladder dysfunction due to frontal lobe lesions. Neurol Med (Tokyo) 1992;36:561-7.
3 Bradley WE. Cystometry and sphincter electromyography. Mayo Clin Proc 1976;51:329-35.

4 Athwal BS, Berkley KJ, Hussain I, et al. Brain responses to changes in bladder volume and urge to void in healthy men. Brain 2001;124:369-77.

5 Sam N, Claus S, Jorgen KI, et al. Cerebral activation during micturition in normal men. Brain 2000;123:781-9.

\section{BOOK REVIEW}

\section{Neuropsychiatry and behavioural neurology explained}

Edited by A J Mitchell. Published by Saunders, London, 2004, £69.00 (hardback), pp 500. ISBN 0-7020-2688-3

This is an ambitious project for a single author; the whole of neuropsychiatry explained using an up to date, evidence based review of the literature, and in a format that is designed to be attractive to read. There are numerous figures, boxes, lists with bullet points, and "clinical pointers" to break up the text.

Although aimed particularly at liaison and old age psychiatrists, this book will have wide appeal and be of interest to neurologists. They will be able to quickly access clinically relevant discussion of the neuropsychiatric sequelae of common neurological disorders. The core sections of the book, on dementia and delirium, neuropsychiatric treatments, and the psychiatric complications of neurological diseases, are excellent. The discussion is practical and to the point. The reader is not stifled with references strewn in the text. They must therefore have confidence in the assertions of the author; I am confident that we are being offered accurate information. But at times the style feels a little pedantic; for example, those of us who dared to believe that alcohol might cause depression are put firmly in our place. Another quibble I have is the value of some of the lists/classifications which were of uncertain provenance. We are, for example, given lists suggesting difference aetiologies for chorea versus athetosis, but some would be sceptical of the value in splitting choreoathetosis. Many classifications are based on neuroanatomical models of neuropsychiatry that need to be treated with caution.

The book strays into biological psychiatry, and a later section is devoted to understanding how neurological disorders result in neuropsychiatric symptoms, but this does cause a problem because some of the discussion of the neuropsychiatric sequelae of a particular disorder may not be found in the index chapter on that disorder, but in this later section. For example, the only discussion of suicide following head injury in the chapter on head injury is a single misleading sentence indicating that suicide accounts for $10 \%$ of head injury deaths. Yet, easily missed, 300 pages later, in the chapter on the neurological origins of suicide, is a more complete account of the relationship.

Overall, however, this book is a significant achievement. A large amount of material has been made readily accessible. There are no lacunae and the length of discussion of each disorder is proportionate to its importance. The book is to be trusted and recommended One interesting innovation is a list of support groups and useful websites in the appendix. Neurologists and psychiatrists and their trainees have good reason to buy this book.

S Fleminger

\section{CORRECTIONS}

doi: 10.1136/jnnp.2004.047118corr l

Wood J M, Worringham C, Kerr G, et al Quantitative assessment of driving performance in Parkinson's disease ( $J$ Neurol Neurosurg Psychiatry 2005; 76:176-80). SD instead of SE was inadvertently inserted during the publication process in tables 2,3 , and 4 . The $\mathrm{p}$ values are unaffected.

doi: 10.1136/jnnp.2003.029389corr l

Barber PA, Demchuk AM, Hill MD, et al. The Probability of middle cerebral artery MRA flow signal abnormality with quantified CT ischaemic change: targets for future therapeutic studies ( $J$ Neurol Neurosurg Psychiatry 2004;75:1426-30). The following errors appeared in this article:

(1) The median CT ASPECTS and DWI ASPECTS quoted in the article were both 8. These are incorrect and should be CT ASPECTS 9 and DWI ASPECTS 8 ;

(2) Sixty-six per cent (95\% CI $0.56-0.75)$ of the patients had CT ischaemic change, while $81 \%$ (95\% CI $0.72-0.88)$ of the DWI scans identified areas of hyperintense signal (not $67 \%$ and $79 \%$ quoted in the article);

(3) In figure 2 the numbers in parentheses on the $\mathrm{x}$ axis were incorrect. The correct numbers for each ASPECTS value are 10 (34), $9(21), 8(12), 7(11), 6(12)$, and 5 (10). 